\title{
Initial Subcellular Responses of Susceptible and Resistant Soybeans Infected with the Soybean Cyst Nematode
}

\author{
Young Ho Kim ${ }^{1 *}$, Kyung Soo Kim² and Robert D. Riggs ${ }^{2}$ \\ ${ }^{1}$ Department of Agricultural Biotechnology, Seoul National University, Seoul 151-921, Korea \\ ${ }^{2}$ Department of Plant Pathology, University of Arkansas, Fayetteville, AR 72701, USA \\ (Received on April 29, 2012; Revised on July 4, 2012; Accepted on July 10, 2012)
}

Initial subcellular responses in susceptible (PI 274420) and resistant (cv. Hartwig) soybeans infected with the soybean cyst nematode (SCN) were examined 2 and 4 days after inoculation (DAI). Subcellular features common to both soybeans at 2 DAI included hypertrophied initial syncytial cells (ISCs) and syncytium-component cells (SCs) with a dense cytoplasm containing proliferated rough and smooth endoplasmic reticulum (RER and SER), a hypertrophied nucleolus, and reduced vacuoles, suggesting that the nematode-infected cells were dedifferentiated. In the resistant soybean, a striking initial subcellular difference from the susceptible soybean was the dilation of the RER, indicating ER dysfunction and leading to cell death. This disturbed nematode feeding, as evidenced by disrupted feeding tubes. In PI 274420, the ISC cytoplasm was depleted, with the exception of ER membranes, at 4 DAI, while the SC cytoplasm was dense with proliferation of starch-containing plastids around multiple nuclei that might be derived from the congregation of nuclei in the neighboring SCs and in part by nuclear division without cytokinesis. In cv. Hartwig, syncytia were necrotized with secondary cell wall thickening outside the plasma membrane and an extremely dense cytoplasm containing a nucleus with an electron-lucent nucleolus, accompanied by the proliferation of closely stacked parallel RER and ribosomes. These results suggest that syncytia develop continuously in PI 274420 to produce and store nutritional substances in SCs, providing for the nematode through ISC until maturation, but in cv. Hartwig, syncytia degenerate early due to excessive metabolism, blocking nematode feeding and cytoplasmic connections with adjacent intact cells.

Keywords : ER dysfunction, soybean, soybean cyst nematode, subcellular responses, syncytium

\footnotetext{
*Corresponding author.

Phone) +82-2-880-4675, FAX) +82-2-873-2317

E-mail)yhokim@snu.ac.kr
}

The soybean cyst nematode (SCN), Heterodera glycines Ichinohe, is distributed globally wherever the soybean (Glycine max Merr.) grows, and is one of the most important pests in soybean production (Wrather et al., 2001). The pathogenicity of SCN is governed by the formation of syncytia in the host plant root as a feeding site, the development of which is directly related to nematode growth and development (Kim and Riggs, 1992; Kim et al., 1986, 1999).

The syncytium is a group of cells, the cytoplasms of which are contiguous due to cell wall dissolution; this provides nutrients for the infecting nematode. The syncytium develops continuously until SCN maturation in susceptible soybeans, while it degenerates early in soybeans resistant to $\mathrm{SCN}$, resulting in poor growth and premature death of the infecting nematodes (Kim et al., 1987, 1999). Syncytium degeneration in resistant soybean cultivars infected with SCN may be a result of the expression of inheritable resistance genes related to hypersensitive responses and/or nuclear degeneration in syncytial cells. The characteristic subcellular responses of soybeans susceptible and resistant to SCN have been well documented, which include syncytium necrosis, secondary cell wall thickenings and nuclear degeneration that are differentiated based on the combinations of SCN races and soybean lines (Kim et al., 2010).

When SCN infects soybean, syncytia are formed from cortical, pericycle, endodermis, xylem, and phloem parenchyma cells (Jones and Dropkin, 1975). Endo (1964) and Gipson et al. (1971) observed syncytium formation within 1 day and $42 \mathrm{~h}$ after inoculation, respectively. In the former work, the cells penetrated by the nematode stylet exhibited cellular hypertrophy, cell wall dissolution, and clumping of nuclei from contiguous cells. The latter study revealed the structural changes caused by SCN infection, such as a decreased vacuole volume and increased cytoplasm and endoplasmic reticulum. In contrast, in SCN-resistant soybeans, two structural modifications occur: formation of necrotic layers accompanying secondary cell wall thickening around syncytial cells, and nuclear degeneration in syncytial cells leading to eventual cell death (Kim et al., 1987). 
Although the structural and subcellular modifications related to the susceptible and resistant responses of soybean root cells have been well documented, there is little information regarding the initial subcellular features that result in differentiation of susceptible and resistant responses. Therefore, the present study investigated the initial subcellular responses of susceptible and resistant soybeans infected with SCN.

\section{Materials and Methods}

Plants and nematode inoculum. SCN race 3 (R3) and susceptible (PI 274420) and resistant (cv. Hartwig) soybeans were used. SCN R3 reared in a greenhouse on cv. Lee 71 was used. Cysts were harvested from infected soybean roots, crushed in distilled water using a glass rod under a stereomicroscope, and placed on Baermann funnels for 3 days to allow second-stage SCN juveniles (J2) to hatch from the eggs. Soybean seeds of PI 274420 and cv. Hartwig were sown and germinated in vermiculite, and their seedlings at the cotyledon stage were transplanted into sterilized river sand in clay pots of $7.5 \mathrm{~cm}$ diameter. Two days later, the rhizosphere of plants were inoculated with $\sim 1,000 \mathrm{~J} 2$ SCN. One day later, roots were uprooted and rinsed in tap water to remove non-penetrating nematodes, and were transplanted into fresh, sterilized soil to synchronize nematode penetration time. SCN-infected soybean plants were cultured at $23-28^{\circ} \mathrm{C}$ in a greenhouse.

Electron microscopy. Two and four days after inoculation (DAI), whole soybean roots infected with $\mathrm{SCN}$ were washed free of sand, and root segments were fixed with
Karnovsky's fixative in $0.05 \mathrm{M}$ cacodylate buffer ( $\mathrm{pH} 7.0$ ) for $2 \mathrm{~h}$, and post-fixed in $1 \%$ osmium tetroxide in the same buffer for $2 \mathrm{~h}$. Then the root samples were rinsed in distilled water and dehydrated in a graded ethanol series $(30 \%, 50 \%$, $70 \%, 95 \%$, and $100 \%$ ethanol for 10 min each; three rinses in $100 \%$ ethanol), further dehydrated in propylene oxide twice (for 10 min each), and embedded in Spurr's epoxy resin (Spurr, 1969). Ultra-thin sections (approximately 80 $\mathrm{nm}$ thickness) were cut with a diamond knife on an ultramicrotome (MT-X, RMC, Tucson, AZ, USA), mounted on copper grids, and double-stained with $2 \%$ (wt/vol) uranyl acetate and Reynold's lead citrate for $7 \mathrm{~min}$. Sections were examined under a transmission electron microscope (JEM1010, JEOL Ltd., Tokyo, Japan) at an accelerating voltage of $80 \mathrm{kV}$.

\section{Results}

Initial structural characteristics of $\mathrm{SCN}$-infected root cells. Penetration and establishment of SCN juveniles in soybean root tissues were confirmed 2 DAI by the presence of initial syncytial cells (ISCs) of nematode bodies with stylets in susceptible (PI 274420) and resistant (cv. Hartwig) soybeans (Fig. 1). Transverse sections of SCN-susceptible soybean roots infected with SCN showed ISCs and associated syncytium-component cells (SCs) at 2 DAI, which were characterized by hypertrophied parenchyma cells with a dense cytoplasm, a hypertrophied nucleolus in an enlarged nucleus, and a thickened primary cell wall, accompanied by cell wall perforations (dissolutions) between ISCs and SCs (Fig. 1A). As in the susceptible host, some ISCs in the resistant cv. Hartwig exhibited cytopathic

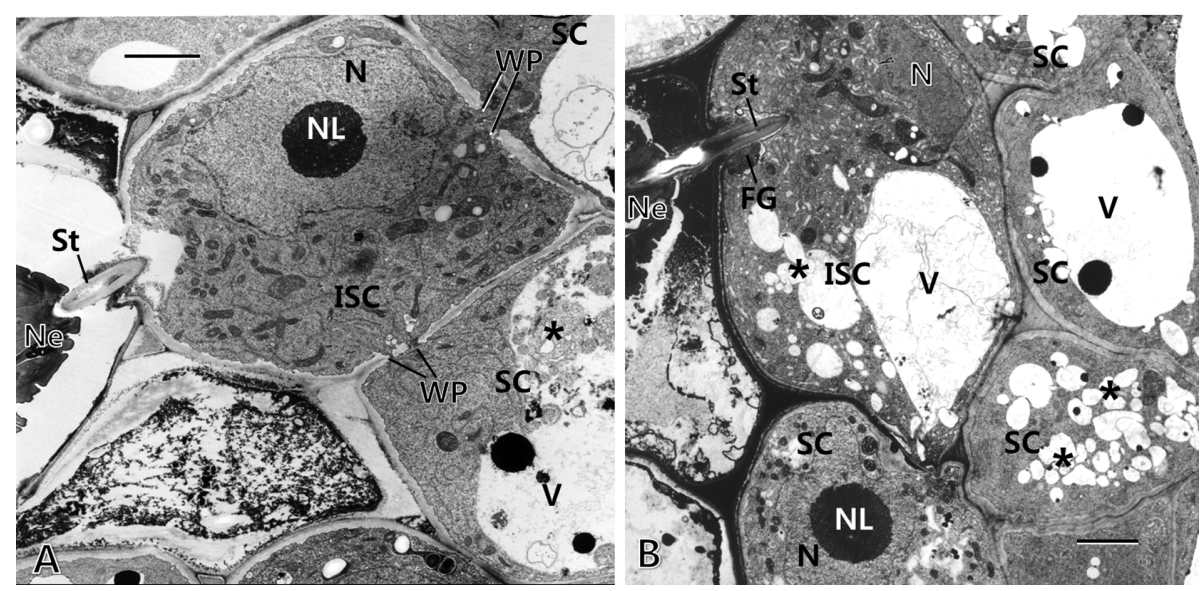

Fig. 1. Electron micrographs of susceptible PI 274420 (A) and resistant cv. Hartwig (B) soybeans infected with soybean cyst nematode race 3 at 2 days after inoculation, showing the formation of initial syncytial cells (ISCs) adjacent to the nematode (Ne) and syncytiumcomponent cells (SCs). The ISCs and SCs in both soybeans are characterized by hypertrophy with a dense cytoplasm and reduced large vacuoles (V) (by cytoplasmic incorporation into the vacuoles [asterisk in A] and/or their breakdown [asterisks in B]) and a meristem-type nucleus (N) containing a hypertrophied and electron-dense nucleolus (NL). Cell wall perforations (WP) between ISCs and SCs were common in the susceptible soybean; however, they were scarce in the resistant soybean. FG $=$ feeding plug. Bars $=2 \mu \mathrm{m}$. 
features characteristic of syncytia, such as hypertrophied cells with a dense cytoplasm and an enlarged nucleus with a hypertrophied nucleolus; however, cell wall perforations between ISCs and SCs were scarce. Vacuoles were reduced by the incorporation of cytoplasmic contents and their breakdown into small vacuoles in ISCs and SCs; however, large central vacuoles remained in parenchymatous cells (Fig. 1A, B).

Subcellular features of initial syncytial cells induced by SCN infection. The dense cytoplasm of ISCs with stylet penetration, as evidenced by feeding tubes in susceptible soybeans, consisted of greatly proliferated membranes, including smooth and rough endoplasmic reticulum (SER and RER), and increased numbers of other organelles, such as mitochondria (Fig. 2A, B). Feeding tubes adjacent to the swollen area of the cell wall (corresponding to the nematode stylet penetration site) appeared to be intact, as they had a clear inside cavity, which functioned as a canal for delivery of nutritional substances from the cytoplasm to the nematode stylet (Fig. 2B). The dense cytoplasm of the stylet penetration site in ISCs in the resistant soybean cV. Hartwig also consisted of greatly proliferated membranes identified as RER due to their association with ribosomes; however, the majority were characteristically dilated (Fig. 2C, D, E). Most mitochondria in the ISCs of cv. Hartwig appeared to be disrobed with few evident cristae (Fig. 2C, D). Feeding tubes at the stylet penetration site in cv. Hartwig were scarce, and appeared to be degenerated with double boundary layers and/or a disrupted inside cavity (Fig. 2D).

\section{Continuous syncytium development in the susceptible} soybean. In the susceptible soybean PI 274420, a wellestablished syncytium was formed 2 DAI, and its ISCs were characterized by a dense cytoplasm with a profound increase in mitochondria and large cell wall perforations between SCs (Fig. 3A). Nuclear division processes were found in SCs, indicated by coalesced chromosomes (probably in the anaphase) (Fig. 3B) and two daughter nuclei (probably in the telophase) (Fig. 3), resulting in nuclear division into two daughter nuclei without cytokinesis (Fig.
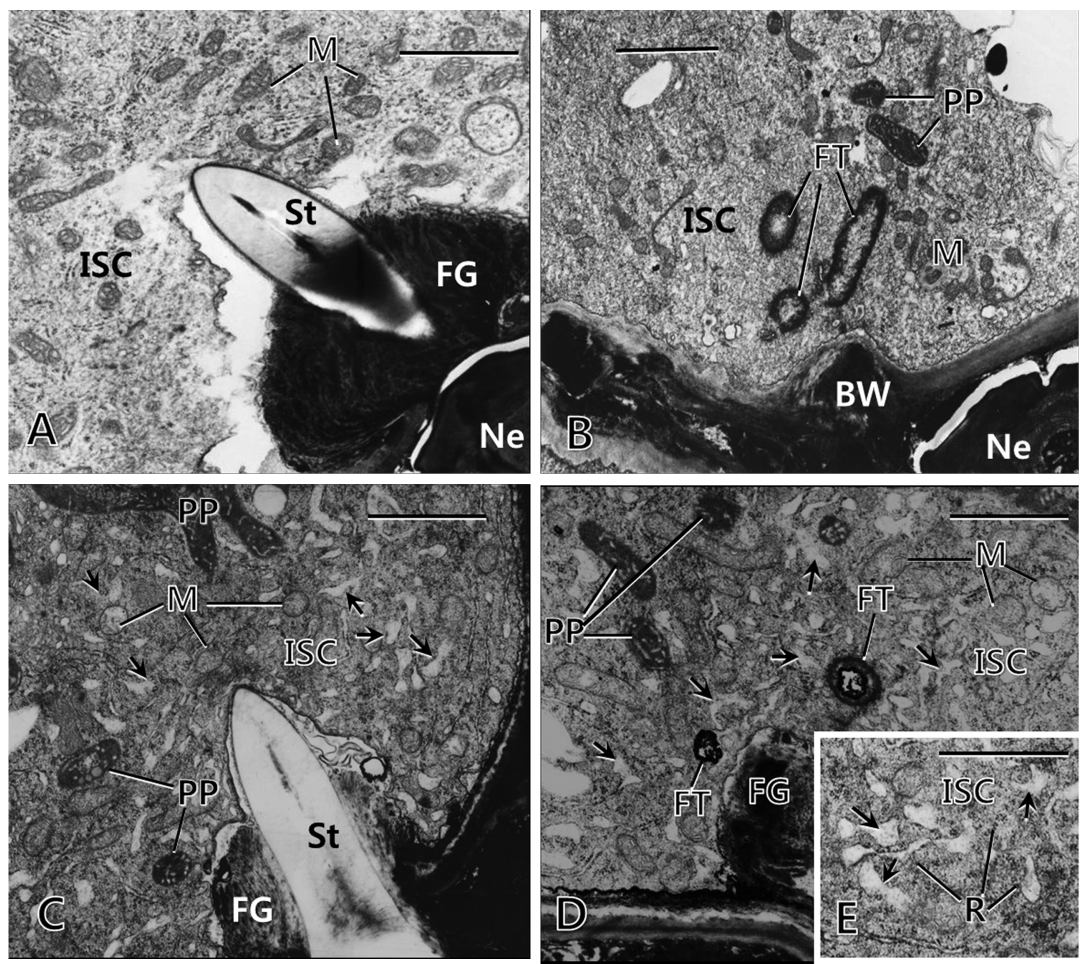

Fig. 2. Electron micrographs of infection sites induced by soybean cyst nematode race 3 in susceptible PI274420 (A, B) and resistant cv. Hartwig (C, D, E) soybeans 2 days after inoculation, showing initial syncytial cells (ISCs) with stylet (St) penetration (evidenced by feeding tubes [FT], a feeding plug [FG], and/or a bulge in the cell wall [BW]). The cytoplasm in the susceptible soybean is characteristically dense, consisting of greatly proliferated membranes, including smooth and rough endoplasmic reticulum and other cell organelles, such as mitochondria (M) and proplastids (PP). The cytoplasm in the resistant soybean also shows features characteristic of membrane proliferation; however, unlike the susceptible host, the cisternae of the rough endoplasmic reticulum (shown to be attached with ribosomes [R] in [E]) were characteristically dilated (arrows). FTs and some $\mathrm{M}$ in the resistant soybean appeared to be degenerated, showing a disrupted inside cavity with double boundary layers and depleted cristae, respectively. Bars $=2 \mu \mathrm{m}$. 

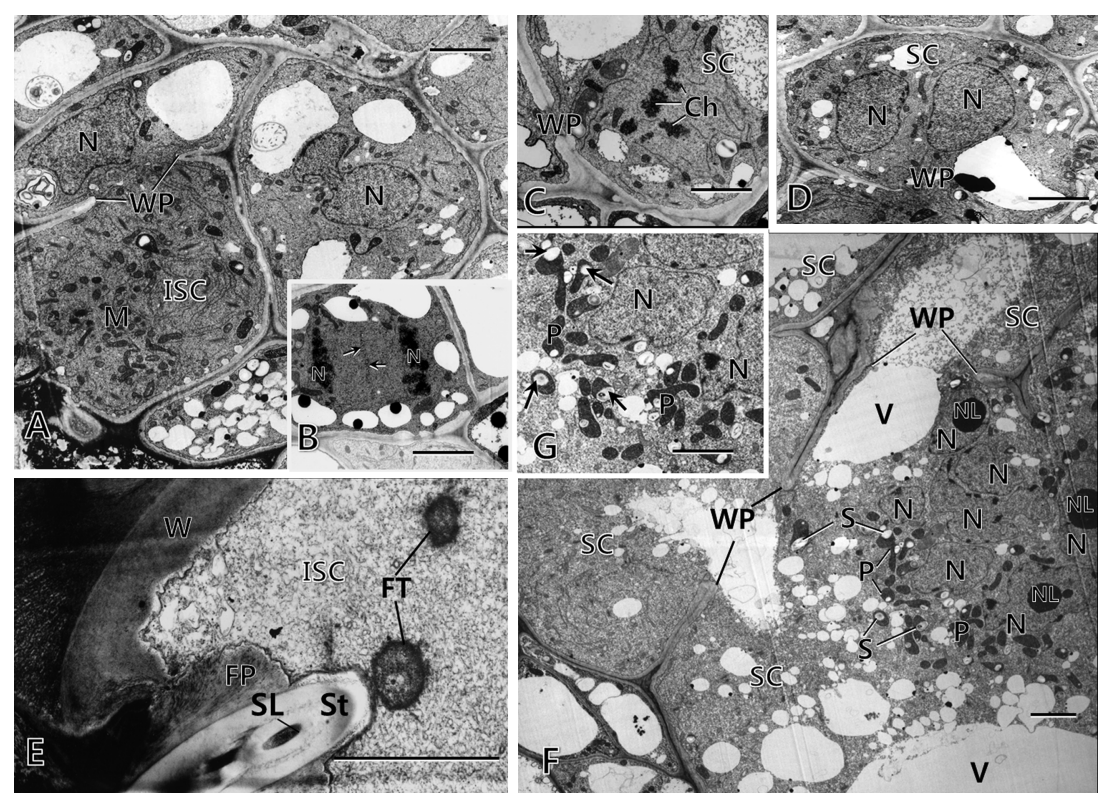

Fig. 3. Electron micrographs of infection sites induced by soybean cyst nematode race 3 in the susceptible soybean PI 274420 at 2 (A, B, $\mathrm{C}, \mathrm{D})$ and 4 (E, F, G) days after inoculation (DAI). At 2 DAI, syncytium-component cells (SCs) were characterized by a dense cytoplasm and increased mitochondria (A) and were undergoing nuclear division, as indicated by the two daughter telophase nuclei (N) and cell plates (CP) in (B), coalesced chromosomes (Ch) in (C) (probably in anaphase), and the two divided daughter nuclei (N) in an ISC in (D). At 4 DAI, some ISCs (indicated by the penetration of nematode stylet [St] with stylet lumen [SL] and feeding plug [FG]) had a homogeneously pale cytoplasm around feeding tubes (FTs), consisting of a proliferated membrane system, indicating that other cytoplasmic contents were consumed by the nematode (Ne) in (E). In contrast, in (F), a well-established syncytium had formed at 4 DAI, characterized by large SCs with a dense cytoplasm and cell wall perforations (WP). The SC in the middle shows seven N, some with hypertrophied electron-dense nucleoli (NL), aggregated in an area in the cytoplasm, which originates from component cells but could have resulted from mitotic nuclear division without cytokinesis as shown in (B-D). Plastids (P) containing starch granules ([S] in [F] and [arrow] in [G]) typically proliferated around the aggregated nuclei in (F) and (G). Bars $=2 \mu \mathrm{m}$.

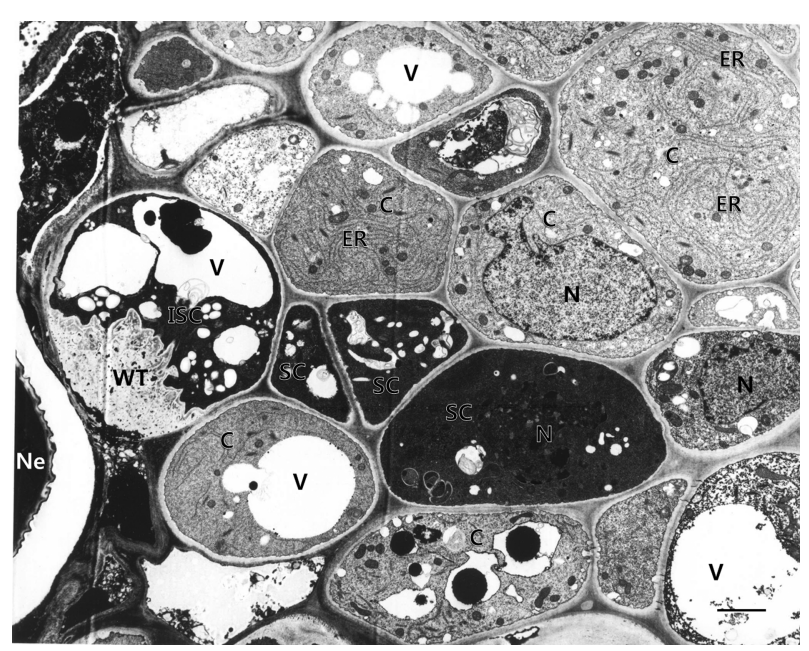

Fig. 4. Electron micrograph of an infection site in the resistant soybean cv. Hartwig infected with soybean cyst nematode race 3 at 2 days after inoculation, showing the formation of extensive abnormal cell wall thickenings (WT) in a callose-like matrix at the site of nematode penetration (Ne) in initial syncytial cells (ISCs). Syncytium-component cells (SCs) adjacent to ISCs were necrotized and had an extremely electron-dense cytoplasm containing a degraded nucleus (N). Cells (C) around ISCs and SCs had a characteristically dense cytoplasm with reduced vacuoles (V) and proliferated endoplasmic reticulum (ER). Bar $=2 \mu \mathrm{m}$.
3D). At 4 DAI, some ISCs exhibited a homogeneously pale cytoplasm consisting of only a proliferated membrane system, indicating that the other cytoplasmic contents that contribute to cytoplasmic density were consumed by the nematode (Fig. 3E). However, SCs formed at 4 DAI in susceptible soybeans retained a dense cytoplasm, in areas in which large vacuoles were in the process of filling up with cytoplasmic contents, becoming dense cytoplasm (Fig. 3F). Also, numerous nuclei with hypertrophied electron-dense nucleoli aggregated in the middle of the SCs, which originated from adjacent $\mathrm{SCs}$ and/or could have resulted from mitosis without cytokinesis, as shown in Figure 3B-D. Numerous plastids containing mostly starch granules congregated around the aggregated nuclei (Fig. 3F, G).

\section{Degeneration of syncytia in the resistant soybean Overall initial structural features of resistant syncytia. In many ISCs in the resistant soybean cv. Hartwig at 2 DAI, extensive abnormal cell wall thickenings consisting of electron-dense particulate deposits in a callose-like matrix developed at the site of nematode penetration (Fig. 4). ISCs and adjacent SCs with these abnormal cell wall thickenings were extremely electron-dense, and appeared to be necrotized}




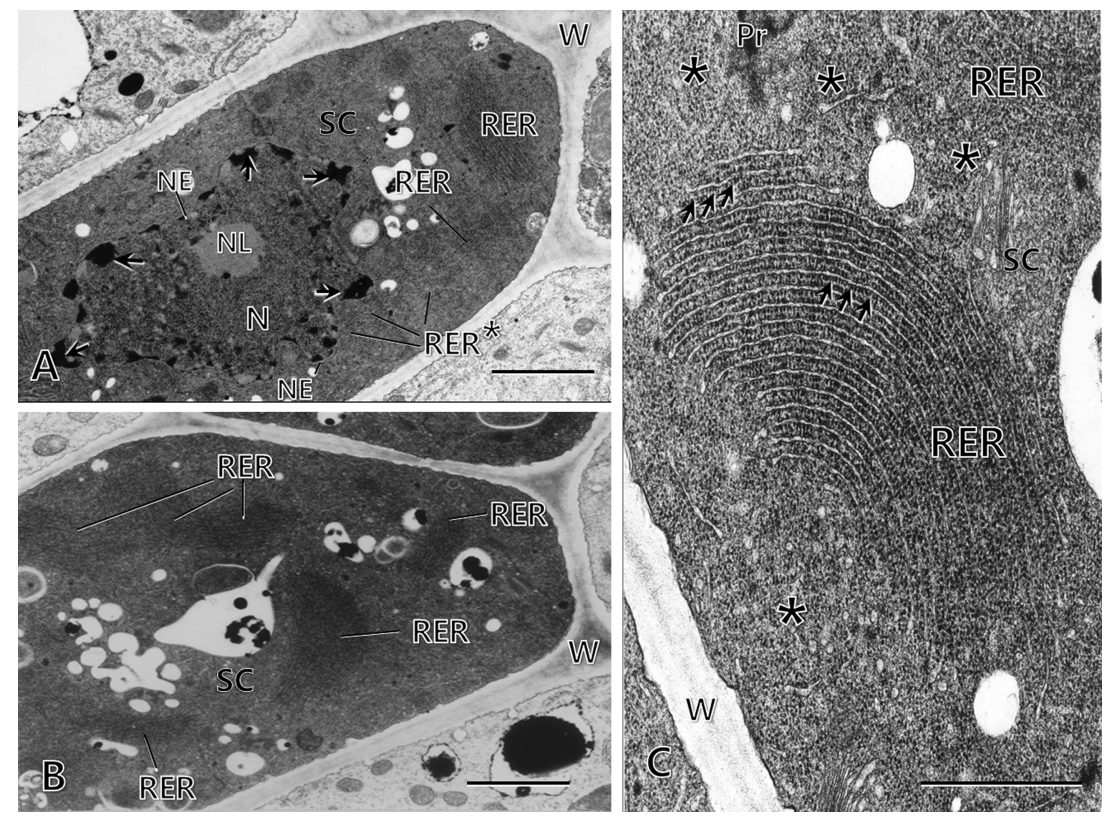

Fig. 5. Electron micrographs of syncytium-component cells (SCs) in the resistant soybean cv. Hartwig infected with soybean cyst nematode race 3 at 2 days after inoculatin. (A) Degraded nucleus (N) with peripheral heterochromatin-like materials (arrows) and an electron-lucent nucleolus (NL) showing the depletion of nucleolar materials and formation of closely stacked rough endoplasmic reticulum (RER) continuous with the nuclear envelope (NE) (indicated by RER*) in SCs. (B) Proliferation of the RER in various locations in SCs. (C) Detailed structural features of the RER showing an unusually regular arrangement of numerous parallel cisternae, confining the intervening cytoplasm to very thin layers containing enormously increased numbers of ribosomes in a linear arrangement attached to ER cisternae (arrows). Proliferated cytoplasmic ribosomes (asterisks) and the probable protein product (Pr) of the ER system were found near the RER in SCs. Bars $=2 \mu \mathrm{m}(\mathrm{A}, \mathrm{B}), 1 \mu \mathrm{m}(\mathrm{C})$.

as a result of hypersensitive responses. All parenchymatous cells surrounding the necrotized syncytial cells exhibited a dense cytoplasm with proliferated endoplasmic reticulum.

\section{Modifications of nuclear and membrane structures in} resistant syncytial cells. Electron micrographs of SCs in the resistant soybean cv. Hartwig infected with SCN R3 at 2 DAI showed a degraded nucleus with heterochromatin materials dispersed peripherally around the nuclear envelope and an electron-lucent nucleolus (Fig. 5A). Closely stacked RER derived from the nucleus were proliferated in an unusually regular arrangement. This was composed of proliferated parallel cisternae that confined the intervening cytoplasm filled with ribosomes, which were attached to the ER cisternae (Fig. 5A, B, C). Also, the cytoplasm surrounding the closely stacked RER was full of ribosomes linked together in an evenly spaced arrangement, resembling polysomes, and contained RER products (Fig. 5C).

Cell wall modifications in the resistant soybean. Electron micrographs of infection sites in the resistant soybean cv. Hartwig infected with SCN R3 at 2 DAI showed ISCs with extensive secondary cell wall thickening in a callose-like matrix at the site of nematode penetration and the formation of cell wall perforations between ISCs and SCs (Fig. 6A). Cell wall perforations were also formed between ISCs and SCs in the resistant soybean (Fig. 6B); however, these were smaller than those in the susceptible soybean. Secondary cell wall thickenings were also formed on SCs bound to both ISCs and other SCs (Fig. 6C, D). Paramural bodies located between the cell wall and plasma membrane were associated with the apposition of secondary wall materials (Fig. 6D). At 4 DAI, ISCs were completely necrotized and had extensively thickened secondary cell walls in a calloselike matrix, in which the lip region of the infecting nematode was embedded completely (Fig. 6E), indicating immobilization of the infecting nematode.

\section{Discussion}

Cyst and root-knot nematodes modify selected plant root cells into complex feeding sites, syncytia and giant cells, respectively, for their growth and development. The nematodes are totally dependent on the formation of these feeding cells for the initiation and maintenance of their parasitism (Davis and Mitchum, 2005; Moon et al., 2008). Retardation of syncytium and giant cell development, and their early degeneration, result in the failure of nematode 


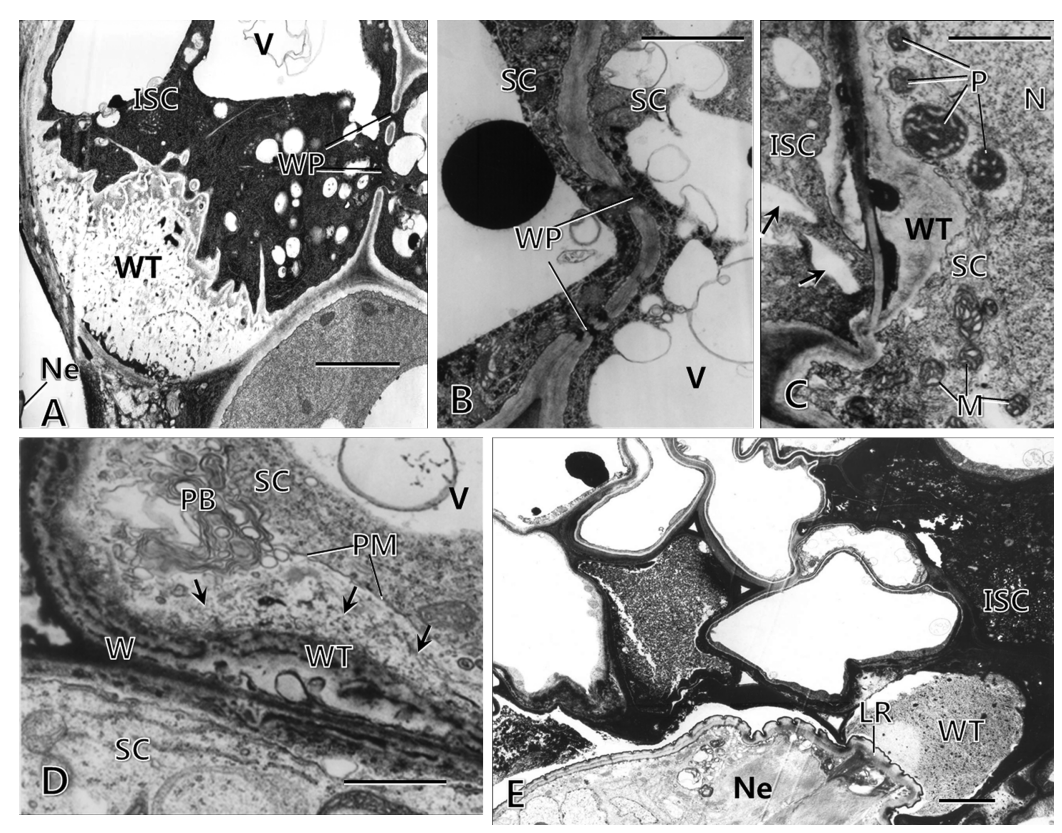

Fig. 6. Electron micrographs of infection sites in the resistant soybean cv. Hartwig infected with soybean cyst nematode race 3 at 2 (A, B, C, D) and 4 (E) days after inoculation (DAI), showing (A) an initial syncytial cell (ISC) with extensive cell wall thickening (WT) in a callose-like matrix at the site of nematode penetration (Ne), and cell wall perforations (WP) between ISCs and syncytium-component cells (SCs), (B) the formation of WP between SCs and adjacent SCs (C, D) the formation of WT in SCs adjacent to ISCs (as indicated by a dilated ER [arrows]), (C) and in SCs adjacent to other SCs (D). In (D), paramural bodies (PB) probably associated with the apposition of secondary wall materials (arrows) on the secondarily thickened cell wall (WT formed between the cell wall [W] and plasma membrane [PM], separating the former from the latter). At 4 DAI (E), ISCs were completely necrotized with the formation of extensive WT in a callose-like matrix in which the lip region (LR) of the infecting nematode $(\mathrm{N})$ was embedded completely. Bars $=2 \mu \mathrm{m}$.

development and parasitism, which is critical for the resistance of plant cultivars and lines to nematodes (Kim et al., 1987; Kim et al., 2010; Moon et al., 2008).

In this study, electron microscopy of the infection sites revealed that structural features of the infected root cells were related to the aspects mentioned above. Well-established syncytia were formed in the susceptible soybean. However, syncytia were degenerated early in the resistant soybean, as indicated by cytoplasmic necrosis and cell wall thickenings, which may represent hypersensitive responses that occur during the early stages of pathogen infection (Agrios, 2005).

The initial features common to both SCN-infected susceptible and resistant soybeans were hypertrophied cells with a dense cytoplasm containing an enlarged nucleus with a hypertrophied nucleolus and increased ER. These are characteristic of the undifferentiated meristematic cells found in zones of the plant where growth can occur (Esau, 1977). However, these modified cells retained large cytoplasmic vacuoles, although they were in the process of cytoplasmic densification by means of vacuole reduction. This suggests that the original cell and tissue types of the nematode infection sites are parenchymatous, but were transformed into a meristematic cell type, which is a process of dedifferentiation in which specialized (differentiated) cells are regressed to unspecialized (undifferentiated) embryonic (meristematic) ones (Esau, 1977). This dedifferentiation usually occurs in the induction of callus for plant regeneration under the influence of the proper ratios of plant hormones, such as auxin and cytokinin, in cell and tissue culture (Sugimoto et al., 2010). Auxin is thought to be the factor most associated with syncytium induction, because exogenous indoleacetic acid causes hypertrophy, hyperplasia, adventitious roots, nuclear division without cytokinesis, and cell wall dissolution (Fan and Maclachlan, 1967). Grunewald et al. (2009) suggested that initiation of syncytia might be triggered by auxin, which is required for the establishment of the nematode feeding sites. In this respect, the initial subcellular changes common to both susceptible and resistant soybeans may not be related to the plant responses that differentiate between susceptibility and resistance to the nematode infection.

The cytoplasmic features of ISCs in the initial infection sites of the susceptible soybean comprised mostly a profound increase in the size of ER and in the number of ribosomes, which were similar superficially to those of the resistant soybean. However, close inspection of the ER structures showed intact and dilated RER in susceptible and resistant ISCs, respectively. Endo (1991) reported similar 
subcellular structures in SCN-infected resistant Bedford soybean root, which were associated with cisternae. In our study, the RER origin of these was suggested by their association with ribosomes. The ER membrane system, which is continuous with the outer layer of the nuclear envelope, is multifunctional with diverse biosynthetic and regulatory roles (Gunning and Steer, 1996). Several innate and exogenous factors can disturb the normal functions of the ER, leading to a conserved cell stress response that is aimed initially at compensating for damage, but can eventually trigger cell death in cases of severe and prolonged ER dysfunction (Xu et al., 2005). Premature death of melanocytes cultured from the vitiligo mouse is caused by an innate defect that affects the structure and presumably the function of the RER, which is dilated (Boissy et al., 1991). The ER also becomes dilated in the cells of resistant chili pepper infected with Colletotrichum gloeosporioides, which likely represents a hypersensitive response leading to programmed cell death and wound periderm formation (Kim et al., 2004). All of these aspects suggest that the ER dilation observed in the present study is part of a hypersensitive response of the resistant soybean to nematode infection. The appearance of disrupted mitochondria in the ISCs of resistant soybean root also suggests premature death (Boissy et al., 1991); this likely hinders nematode feeding, because disrupted feeding tubes were observed in the ISCs of the resistant soybean cv. Hartwig at 2 DAI (Fig. 2D).

In the susceptible soybean, syncytial development was continued by integration of adjacent SCs, leading to multinucleation. With continuing nematode infection, the syncytium expands by integrating more adjacent cells and ultimately incorporates several hundred cells, suggesting that the multi-nucleic state of a syncytium may be derived from the incorporation of the nuclei of incorporated SCs (Grunewald et al., 2009). Also cell wall dissolution and clumping of nuclei from contiguous cells were found in the cells penetrated by the SCN stylet and exhibiting cellular hypertrophy, by which it has been regarded that the syncytium multi-nucleation might result from the aggregation of neighbouring SCs (Endo, 1964). However, we detected nuclear division processes in SCs during syncytium expansion, as evidenced by coalesced chromosomes probably in the anaphase, two daughter nuclei possibly in the telophase, and two divided daughter nuclei, indicating completion of nuclear division (Fig. 3B-D) (Gunning and Steer, 1995). Thus, it is possible that some of the nuclei in a syncytium originate from nuclear divisions without cytokinesis, which represents a novel finding of multinucleation in syncytium formation.

In the susceptible soybean at 4 DAI, the cytoplasm of ISCs with stylet penetration was sparse and depleted, with the exception of ER membrane structures, suggesting that other cytoplasmic contents are consumed by the infecting nematode. However, most SCs adjacent to ISCs contained dense cytoplasm with proliferation of starch-containing plastids around multiple nuclei with hypertrophied electron-dense nucleoli, which is similar to those of ISCs at 2 DAI. This suggests that SCs at 4 DAI remain metabolically active and thus may act as a substitute for ISCs in terms of transportation and secretion of nutrients (Gunning and Steer, 1995), in this case feeding the nematode from SCs via ISCs. Syncytial cells during the later stages of infection exhibited a profound accumulation of plastids around nuclei and along the cell wall of syncytia (Kim et al., 1989). Starch granules in plastids in the final maturation stages of the nematode are dissolved, suggesting the consumption of stored nutritional substances by the nematode (Kim et al., 1999). Considering that plastids are responsible for the storage of products such as starch (Esau, 1977), the nematode-infected cells of the susceptible soybean may also store nutritional substances in SCs early after infection, to provide nutrients for nematode growth and development until maturation.

Syncytium degeneration in the resistant soybean cv. Hartwig at advanced inflection stages in our study were characterized by cellular necrosis and secondary cell wall thickenings, which resembled those found in SCN-resistant soybean PI 437654, the original genetic source of cv. Hartwig (Anand, 1992; Anand et al., 1985; Kim et al. 2010). The infecting nematodes are likely doomed to death due to the degeneration of the syncytia they feed on.

To the best of our knowledge, this is the first report of the proliferation of closely stacked RER with an unusually regular arrangement of parallel cisternae in the necrotized SCs of the resistant soybean (Fig. 5). Numerous ribosomes were distributed along and around the ER cisternae in an evenly spaced arrangement similar to polysomes, and the nucleolus, the cell's ribosome factory, was electron-lucent (indicating the depletion of nucleolar material). This suggests that the RNA metabolism of the nucleolus might have been excessively accelerated, causing depletion of nucleolar materials and production of ribosomes that were released into the cytoplasm and used for protein synthesis and transportation (Hernandez-Verdun et al., 2010). Thus, resistant soybean root cells infected with SCN may exhaust their ability to defeat the infecting nematode, resulting in cell death.

Cytoplasmic connections are broken by separation of the plasma membrane from the cell wall due to the formation of secondary cell wall thickenings that wall off plasmodesmata (Kim et al., 1987; Riggs et al., 1973). Also, paramural bodies between the cell wall and plasma membrane are associated with cell wall appositions that block plasmodesmata at the site of an intact mesophyll cell underlying 
a hypersensitive epidermal cell (An et al., 2007). This suggests that the secondary wall thickenings formed in the resistant SCs block the cytoplasmic connections and thus inhibit the transport of syncytium-inducing material to the surrounding normal cells, leading to the accumulation of toxic materials and the degeneration of the syncytium.

Conclusively, the initial subcellular modifications in SCN-susceptible soybean suggest the existence of intimate plant-nematode relations that allow the infecting nematode to utilize the metabolically active plant root cells for the continuous production and transportation of nutrients until nematode maturation. On the other hand, the subcellular modifications suggest that the SCN-resistant soybean has an organized defense system against the infecting nematode, in which the root cells and tissues struggle to their full capacity, and eventually die. This inhibits nematode feeding and further syncytium development by blocking cytoplasmic connections between SCs and other intact cells through formation of secondary cell wall thickenings.

\section{References}

Agrios, G. N. 2005. Plant Pathology, Fifth edition. Academic Press, New York.

An, Q., van Bel, A. J. E. and Hückelhoven, R. 2007. Do plant cells secrete exosomes derived from multivesicular bodies? Plant Signal Behav. 2:4-7.

Anand, S. C. 1992. Registration of Hartwig soybean. Crop Sci. 32:1069-1070.

Anand, S. C., Wrather, J. A. and Shumway, C. R. 1985. Soybean genotypes with resistance to races of soybean cyst nematode. Crop. Sci. 25:1073-1075.

Boissy, R. E., Beato, K. E. and Nordlund, J. J. 1991. Dilated rough endoplasmic reticulum and premature death in melanocytes cultured from the vitiligo mouse. Am. J. Pathol. 138:15111525.

Davis, E. L. and Mitchum, M. G. 2005. Nematodes, sophisticated parasites of legumes. Plant Physiol. 137:1182-1188.

Endo, B. Y. 1964. Penetration and development of Heterodera glycines in soybean roots and related anatomical changes. Phytopathology 54:79-88.

Endo, B. Y. 1991. Ultrastructure of initial responses of susceptible and resistant soybean roots to infection by Heterodera glycines. Rev. Nematol. 14:73-94.

Esau, K. 1977. Anatomy of Seed Plants, 2nd Edition. John Wiley \& Sons, New York, USA. pp. 550.

Fan, D.-F. and Maclachlan, G. A. 1967. Massive synthesis of ribonucleic acid and cellulase in the pea epicotyl in response to indoleacetic acid, with and without concurrent cell division. Plant Physiol. 42:1114-1122.

Gipson, I., Kim, K. S. and Riggs. R. D. 1971. An ultrastructural study of syncytium development in soybean roots infected with Heterodera glycines. Phytopathology 61:347-353.

Grunewald, W., van Noorden, G., Van Isterdael, G., Beeckman, T.,
Gheysen, G. and Matghesius, U. 2009. Manipulation of auxin transport in plant roots during Rhizobium symbiosis and nematode parasitism. Plant Cell 21:2559-2562.

Gunning, B. E. S. and Steer, M. W. 1996. Plant Cell BiologyStructure and Function. Jones and Bartlett Publishers, Sudbury, MA, USA.

Hernandez-Verdun, D., Roussel, P., Thiry, M., Sirri, V. and Lafontaine, D. L. J. 2010. The nucleolus: structure/function relationship in RNA metabolism. WIREs RNA 1:415-431.

Jones, M. G. K. and Dropkin, V. H. 1975. Cellular alterations induced in soybean roots by three endoparasitic nematodes. Physiol. Plant Pathol. 5:119-124.

Kim, K.-H., Yoon, J.-B., Park, H.-G., Park, E. W. and Kim, Y. H. 2004. Structural modifications and programmed cell death of chili pepper fruit related to resistance responses to Colletotrichum gloeosporioides infection. Phytopathology 94:12951304.

Kim, K. S. and Riggs, R. D. 1992. Cytopathological reactions of resistant soybean plants to nematode invasion. In: Biology and management of the soybean cyst nematode, ed. by R. D. Riggs and J. A. Wrather, pp. 157-168. APS Press, St. Paul, MN, USA.

Kim, Y. H., Kim, K. S. and Riggs, R. D. 1986. Morphological characteristics of syncytia in susceptible hosts infected by the soybean cyst nematode. Phytopathology 76:913-917.

Kim, Y. H., Kim, K. S. and Riggs, R. D. 2010. Differential subcellular responses in resistance soybeans infected with soybean cyst nematode races. Plant Pathology J. 26:154-158.

Kim, Y. H., Riggs, R. D. and Kim, K. S. 1987. Structural changes associated with resistance of soybean to Heterodera glycines. J. Nematol. 19:177-187.

Kim, Y. H., Riggs, R. D. and Kim, K. S. 1989. Common structural features of syncytia in selected susceptible hosts infected with the soybean cyst nematode. Korean J. Plant Pathol. 5:337-343.

Kim, Y. H., Riggs, R. D. and Kim, K. S. 1999. Heterodera glycines-induced syncytium structures related to the nematode growth and reproduction in susceptible soybean cultivars. Plant Pathology J. 15:1-7.

Moon, H. S., Khan, Z., Kim, S. G., Son, S.-H. and Kim, Y. H. 2010. Biological and structural mechanisms of disease development and resistance in chili pepper infected with the rootknot nematode. Plant Pathology. J. 26:149-153.

Riggs, R. D., Kim, K. S. and Gipson, I. 1973. Ultrastructural changes in Peking soybeans infected with Heterodera glycines. Phytopathology 63:76-84.

Spurr, A. R. 1969. A low-viscosity epoxy resin embedding medium for electron microscopy. J. Ultrastructr. Res. 26:31-43.

Sugimoto, K., Gordon, S. P. and Meyerowitz, E. M. Regeneration in plants and animals: dedifferentiation, transdifferentiation, or just differentiation? Trends Cell Biol. 21:212-218.

Wrather, J. A., Anderson, T. R., Arsyad, D. M., Tan, Y., Ploper, L. D., Porta-Puglia, A., Ram, H. H. and Yorinori, J. T. 2001. Soybean disease loss estimates for the top ten soybean producing countries in 1998. Can. J. Plant Pathol. 23:115-121.

Xu, C., Baily Maitgre, B. and Reed, J. C. 2005. Endoplasmic reticulum stress: cell life and death decisions. J. Clin. Invest. 115:2656-2664. 